Available online on 15.05.2020 at http://jddtonline.info
Open Access to Pharmaceutical and Medical Research
unrestricted non-commercial use, provided the original work is properly cited

Open $\odot$ Access

Review Article

\title{
Role of Micro Emulsion Based In-Situ Gelling System of Fluoroquinolone for Treatment of Posterior Segment Eye Diseases (PSED)
}

\author{
Eram Fatima *1, Dr. Vivek ${ }^{2}$ \\ Department of Pharmacy, IIMT College of Pharmacy, Greater Noida, G.B Nagar, U.P., India
}

\begin{abstract}
The in situ drug delivery system and colloidal formulation like micro emulsion has potential to use in ocular delivery. Micro emulsion provides better permeation of drug through the membrane and provides improved bioavailability. The in situ drug delivery system decreases pre-corneal drainage, increase the contact time of formulation with eye and prolong the release in ocular tissues. Again, in situ gelling system has advantage of delivering accurate and reproducible quantities each time, against any already gelled formulations. To combine the benefits of these two dosage forms, micro emulsion based in situ gelling system can be developed as a novel vehicle for ophthalmic drug delivery. Endophthalmitis is an infection of intraocular fluids like vitreous humor and ocular tissues. To combat the disease, the formulation which provides sufficient concentration in posterior segment eye diseases (PSED) is required.
\end{abstract}

Keywords: Posterior segment eye diseases, micro emulsion, drug delivery system, ocular, formulation.

Article Info: Received 24 Feb 2020; Review Completed 20 April 2020; Accepted 29 April 2020; Available online 15 May 2020

Cite this article as:

Fatima E, Vivek, Role of Micro Emulsion Based In-Situ Gelling System of Fluoroquinolone for Treatment of Posterior Segment Eye Diseases (PSED), Journal of Drug Delivery and Therapeutics. 2020; 10(3):265-272

http://dx.doi.org/10.22270/jddt.v10i3.4079

*Address for Correspondence:

Ms. Eram Fatima* (Research scholar), IIMT College of Pharmacy, Knowledge Park III, Greater Noida, G.B Nagar Greater Noida, G.B Nagar, Uttar Pradesh 201308

\section{Introduction}

\section{Common Ocular Disorders}

The anterior segment of eye consists of the front one-third of the eye that includes; pupil, cornea, iris, ciliary body, aqueous humor, and lens and the posterior segment of the eye consists of the back two-thirds of the eye that includes vitreous humor, retina, choroid, macula, and optic nerve. Accordingly, the common ocular diseases can be classified as anterior segment disorders and posterior segment disorder 1.

\section{Anterior segment disorders}

- Conjunctivitis \& Scleral Diseases

- Corneal Disorder

- Eyelid \& Tearing disorder

- Glaucoma

- Cataract etc.

\section{Posterior segment disorders}

- Optic Nerve disorders

- Choroidal disorders

- Uvetitis

\section{Introduction to Endopthalmitis}

Endopthalmitis is an infection inside the eye. It is very uncommon disease. It is caused by organisms that may enter the eye through a surgical incision [1] or an injury to the eyeball or, very rarely, has travelled through bloodstream into the eye. Possible causes of infection in the bloodstream are intravenous drug use, an abscess (a collection of pus), and skin ulcers, infections such as pneumonia or sepsis, or surgery anywhere in the body. Bacteria are mainly responsible for the infection but it may also occur due to fungi or protozoa. Viruses may causes an eye infection, but this type of infection does not include under the classification of Endophthalmitis 2 .

\section{Common types of Endophthalmitis}

Two broad categories of endophthalmitis are Infectious Endophthalmitis and Non-infectious Endophthalmitis 3 . The infectious endophthalmitis are further distinguished into endogenous and exogenous infections. Exogenous infection results from introduction of organisms into the eye through a surgical or traumatic penetrating wound. Endogenous (or metastatic) infection is caused by organisms that enter the eye via the bloodstream. Both 
categories of infection are extremely serious, threatening blindness and even loss of the globe.

\section{Causative organisms of Endopthalmitis}

The term endophthalmitis is referred as bacterial, fungal or protozoal infection which involves intraocular tissues (retina, uveal tract, or lens) or ocular fluids (vitreous or aqueous). A bacterium isolated from post-operative Endophthalmitis contains about $94.2 \%$ of gram positive bacteria (70\% due to Staphylococcus epidermidis). Other commonly found pathogens are Staphylococcus aureus, Streptococcus pneumoniae, Streptococcus pyogenes, propionibacterium acnes, Haemophilus influenza, E coli,
Bacillus cereus, Neisseria gonorrhoea, and Proteus mirabilis 4 .

\section{Signs and Symptoms}

Symptoms of endophthalmitis include pain, redness, high sensitivity to bright light, and partial or almost complete loss of vision. Sometimes these may be very severe. Other most common signs of endophthalmitis are decreased vision, mild to moderate anterior chamber reaction, hypopyon (pus in the eye), vitritis, variable pain, conjunctival hyperaemia, chemosis, lid oedema, corneal oedema, poor fundal glow. Figure shows 78-year-old man's eye with the symptoms of endophthalmitis 5 .

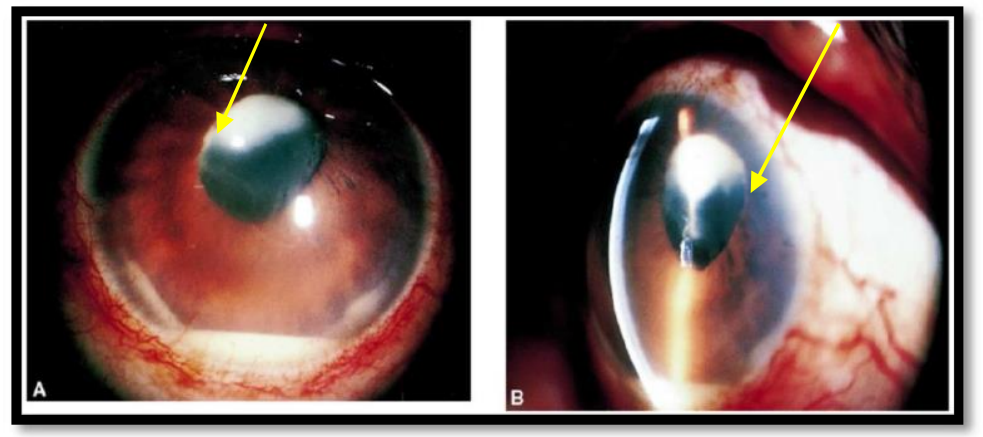

Figure 1: Staphylococcus epidermidischronic endophthalmitis in a 78- year-old man

$A$ : Frontal view. $B$ : Tangential view.

(Courtesy: Survey of Ophthalmology, Michael S. Kresloff et al.)

\section{Detection and Diagnosis}

The diagnosis of endopthalmities is symptomatic. It involves an eye and culture examination. Sometimes antibody or DNA testing also required. For diagnosis, cultures are taken from the aqueous humor and the vitreous humor and plated on sheep blood agar, chocolate agar, thioglycolate broth, and Sabrouraud's dextrose media and smears are treated with Giemsa and Gram staining to found out the responsible organism and also to found out the drugs which is most active against them 6 .

\section{Drugs used in Endophthalmitis}

For an ocular antibiotic selection, one should evaluate four main characteristics of antibiotic. First, the drug must be able to effectively penetrate into the intraocular tissues. Second, the drug must be of adequate potency, which, in the case of antibiotics, is measured by the mean inhibitory concentration (MIC). Third, there must be minimal bacterial resistance to the drug. Finally, the drug should be able to rapidly eradicate bacteria, before infection is able to set in. Generally, the broad spectrum antibiotic is required for the severe vision-threatening infections such as Endophthalmitis. The fourth-generation fluoroquinolones such as Ciprofloxacin (Vigamox) and Gatifloxacin (Zymar), can provide better coverage for gram-positive and resistant organisms than the thirdgeneration fluoroquinolone such as levofloxacin (Quixin, Iquix), and the second-generation fluoroquinolones such as Ciprofloxacin (Ciloxan) and Ofloxacin (Ocuflox). Ciprofloxacin and Gatifloxacin provides improved spectrum of activity as well as increased penetration into ocular tissues, and delayed propensity to the development of bacterial antibiotic resistance. Considering photosensitivity of drug, Ciprofloxacin and Gatifloxacin are not photosensitive like other halogenated fluoroquinolones (i.e. Clinafloxacin, Sitafloxacin, and Lomefloxacin). Ciprofloxacin and Gatifloxacin possess methoxy group at the C8 position; instead of fluorine which is associated with photosensitivity. For these reasons Ciprofloxacin or Gatifloxacin can be best option for the treatment of endophthalmitis 7.

\section{Treatment options ${ }^{8}$}

\section{Anti microbial therapy}

- Intra-vitreal

- Topical

- Peribulbar or Intra cameral

- Systemic

\section{Anti-inflammatory therapy (NSAIDS \& corticosteroids)}

- Intra-vitreal

- Topical

- Systemic

\section{Supportive therapy}

- Anti glaucoma medication

- Vitamins

\section{Therapeutic challenges}

The factors to be considering during treatment of endopthalmities are delicate nature of the interior of the eye and the anatomic barriers. The key anatomic barriers that prevent adequate treatment of endophthalmitis are the inner and outer blood-retinal barrier and the blood- 
aqueous humor barrier, collectively called the bloodocular barrier. The blood-ocular barrier consists of tight junctions between the endothelial cells and basement membrane of retinal capillaries and retinal pericytes. The role of barrier is to protect the interior of the eye from assault by cells, macromolecules, and drugs \& prevents the entrance and subsequent activity of most systemic antimicrobial and anti- inflammatory drugs. Direct injection of drugs into the vitreous humorcan bypass the intraocular barriers. Photoreceptors and other cells of the retina are extremely sensitive to insult, and high doses of antimicrobial agents required to eradicate the bacteria from eye may induce toxic effects on the retina and it may potentially disrupting the biochemical pathways necessary for vision. For the treatment of some vitreoretinal diseases, systemic administration of drugs has been used. But the literature said that after systemic administration, very small amount of drugs could reach the eye. Therefore to obtain a therapeutic level of drug concentration in the eye, a large amount of systemic dose is required.

\section{Ocular Drug Delivery}

\section{Anatomical and Physiological considerations in topical ocular delivery}

It is the tear film which provides corneal transparency and good visual function that require for uniform eye surface. The tear film covers and lubricates the cornea and the external globe. It is the first structure encountered by topically applied drugs and it shows the trilaminar structure of the tear film. Mucous layer is attached to the glycocalix of the corneal/conjunctival surface and it consists mainly of glycoproteins. It is responsible for the wetting of corneal and conjunctival epithelium and also for the stability of tear film. The middle aqueous layer composed of about $98 \%$ of the tear film. It is also composed of water, electrolytes, and various proteins such as lipocalin, lysozyme, and lactoferrin. The outermost lipid layer consists of sterol esters, triacylglycerols, and phospholipids. The main role of this layer is to prevent the evaporation of the tear fluid and is spread over the aqueous layer during blinking 9 .

\section{Nasolacrimal drainage system}

The lacrimal gland is responsible for most of the tear fluid secretion. Secreted fluid is spread over the surface of the cornea during blinking and ends up in the puncta when the upper eye lid approaches the lower lid. The blinking process creates a suction mechanism which results in tears flowing through the lacrimal canaliculi into the lacrimal sac. Fluid from the lacrimal sac then drains into the $12 \mathrm{~mm}$ long nasolacrimal duct, which empties into the inferior nasal passage. This passage is a highly vascular area and is responsible for most of the systemic drug absorption and subsequent systemic side effects of topically administered drug 10 .

\section{Transport barriers in the eye}

Topical administration is the most common route for ocular drug delivery. Consequently, the cornea, conjunctiva, and sclera form the most essential barriers for drug penetration into the intraocular tissues.

\section{Cornea:}

The cornea is composed of five layers: It is considered to be the main pathway for ocular penetration of topically applied drugs. However, due to its unique structure, with the hydrophilic stroma sandwiched between the highly lipophilic epithelium and the less lipophilic endothelium, the penetration of compounds through the cornea depends on their n-octanol - water partition coefficient. Only drugs with a partition coefficient between 10-100 that show both lipid and water-soluble properties can readily pass through cornea 11 .

\section{Conjunctiva:}

The conjunctiva is a thin and vascular mucous membrane consisting of two to three layers of epithelial cells overlying a loose, highly vascular connective tissue. The tight junctions present on the apical surface of the epithelium act as the main barrier for drug penetration (molecules $>20,000 \mathrm{Da}$ ) across the tissue, although not as tight as the corneal epithelium, which is impermeable to molecules larger than $5000 \mathrm{Da}$. The conjunctiva covers the anterior surface of the globe (bulbar conjunctiva), with the exception of cornea, and is folded at the fornix (fornix conjunctiva) to form the palpebral conjunctiva, which lines the inner surface of the eyelids. The bulbar conjunctiva represents the first barrier for permeation of topically applied drugs via the non-corneal route.

\section{Sclera:}

The sclera is the outermost firm coat of the eye that serves as a protective barrier for the sensitive inner parts. It is composed of the same type of collagen fibers as the corneal stroma. However, the fibers are arranged in an irregular network rather than a lattice pattern, which makes the tissue appear opaque compared to the transparent cornea. The white sclera constitutes the posterior 5/6th of the globe, whereas the transparent cornea comprises the anterior $1 / 6$ th of the globe ${ }^{12}$.

\section{Protective blood - ocular barriers:}

The blood-ocular barriers can be divided into bloodaqueous barrier and blood-retinal barrier. The bloodaqueous barrier is located in anterior part of the eye and is formed by endothelial cells of the blood vessels in iris and non-pigmented cell layer of the ciliary epithelium. It regulates the solute exchange between the blood and the intraocular fluid, preventing unspecific passage of solutes that could influence the transparency of the ocular tissues. The outward movement into the systemic blood circulation is less restricted, allowing especially small and lipophilic drug molecules to enter the uveal blood circulation. These molecules are consequently removed more rapidly from the anterior chamber than larger, hydrophilic molecules, which are eliminated by the aqueous humor turnover only. The blood - retinal barrier can be found in the posterior part of the eye. It prevents toxic molecules, plasma components, and water from entering the retina. It also forms a barrier for passage of systemically administered drugs into the vitreous, typically resulting in only $1-2 \%$ of the drug's plasma concentration in the intraocular tissues 13 .

\section{Ocular drug delivery approaches}

They offer some advantages such as their ease of administration by the patient, ease of preparation, and the low production costs. However, there are also significant disadvantages associated with the use of conventional solutions in particular, including the very short contact time with the ocular surface and the fast nasolacrimal drainage, both leading to a poor bioavailability of the drug. 


\section{Polymeric delivery systems}

Polymeric systems used for ocular drug delivery can be divided into three groups: viscosity enhancing polymers, which simply increase the formulation viscosity, resulting in decreased lacrimal drainage and enhanced bioavailability; mucoadhesive polymers, which interact with the ocular mucin, therefore increasing the contact time with the ocular tissues; and in situ gelling polymers, which undergo sol-to-gel phase transition upon exposure to the physiological conditions present in the eye 14 .

\section{Viscosity -enhancing polymers:}

In order to reduce the lacrimal clearance (drainage) of ophthalmic solutions, various polymers have been added to increase the viscosity of conventional eye drops, prolong pre-corneal contact time, and subsequently improve ocular bioavailability of the drug. Among the range of hydrophilic polymers investigated in the area of ocular drug delivery are polyvinyl alcohol (PVA) and polyvinyl pyrrolidone (PVP), cellulose derivates such as methylcellulose (MC), polysaccharides, poly methylvinyl ether maleic anhydride and polyacrylic acids (carbopols). Typically, these polymers are high molecular weight molecules $(5,000-100,000 \mathrm{Da})$, that cannot cross biological membranes 15 .

\section{Mucoadhesive polymers:}

Bioadhesion refers to the attachment of a drug molecule or a delivery system to a specific biological tissue by means of interfacial forces. If the surface of the tissue is covered by a mucin film, as is the case for the external globe, it is more commonly referred to as mucoadhesion. Cationic polymers (like chitosan), which are able to interact with the negative sialic acid residues of the mucin, would probably show better mucoadhesive properties than anionic or neutral polymers. The most commonly used bioadhesives are macromolecular hydrocolloids with numerous hydrophilic functional groups capable of forming hydrogen bonds (such as carboxyl, hydroxyl, amide, and sulfate groups). Hyaluronic acid is also most promising mucoadhesive polymer in ocular drug delivery 16.

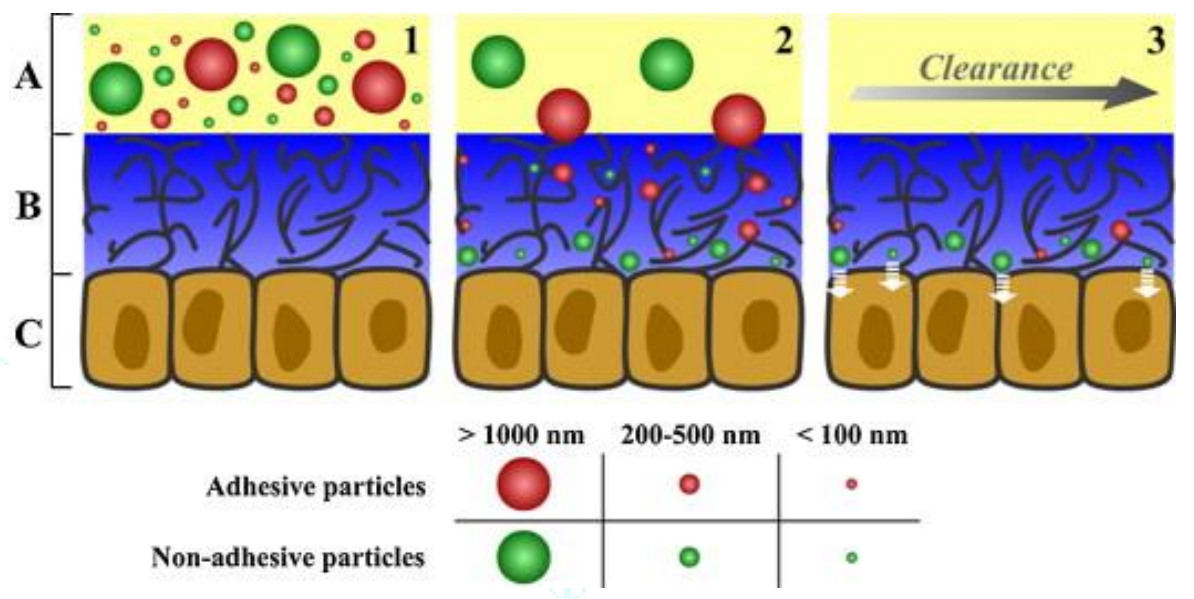

Figure 2: Mucoadhesive polymers in the design of nano-drug delivery systems

\section{Role of Micro emulsion based in situ gelling system}

The use of preformed gels still has drawbacks that can limit their interest for ophthalmic drug delivery. They do not allow accurate and reproducible administration of drugs and after administration; they often produce blurred vision, crusting of eyelids, and lacrimation. In situ gelling approach amalgamates the advantages of both solutions and gels, such as accuracy and facility of administration of the former and prolonged residence time of the latter. Thus in situ gels can be instilled as eye drops and undergo an immediate gelation when in contact with the eye. In situ forming gels are liquid upon instillation and undergo phase transition in the ocular cul-de-sac to form viscoelastic gel and this provides a response to environmental changes ${ }^{17}$.

\section{Colloidal Drug Delivery System}

Colloidal carriers are small particulate systems ranging in size from 100 to $400 \mathrm{~nm}$. As they are usually suspended in an aqueous solution, they can easily be administered as eye drops, thus avoiding the potential discomfort resulting from bigger particles present in ocular suspensions or from viscous/sticky preparations ${ }^{18}$. Most efforts in ophthalmic drugedelivery have been made with the aim of increasing the corneal penetration of the drug ${ }^{19}$.

Colloidal particles are preferably taken up by the corneal epithelium via endocytosis. Cornea acts as a drug reservoir, slowly releasing the active compound present in the colloidal delivery system to the surrounding ocular tissues.

\section{Nanoparticles:}

Nanoparticles are defined as submicrometer sized polymeric colloidal particles ranging from 10 to $1000 \mathrm{~nm}$ in which the drug can be dissolved, entrapped, encapsulated, or adsorbed. Depending on the preparation process, nanospheres or nanocapsules can be obtained. Nanospheres have a matrix like structure where the drug can either be adsorbed at the surface of the particle or be dispersed/dissolved in the matrix. Nanocapsules, on the other hand, consist of a polymer shell and a core, where the drug can either be dissolved in the inner core or be adsorbed onto the surface 20.

The most commonly used biodegradable polymers in the preparation of nanoparticulate systems for ocular drug delivery are poly-alkylcyanoacrylates, poly- -caprolactone, and polylactic-co-glycolic acid copolymers. The major limiting issues for the development of nanoparticles include the control of particle size and drug release rate as 
well as the formulation stability. There is only one microparticulate ocular delivery system in the market, Betoptic S $0.25 \%$. It is obtained by binding of betaxolol to ion exchange resin particles and is found to be bioequivalent to the Betoptic $0.5 \%$ solution in lowering the intraocular pressure ${ }^{21}$.

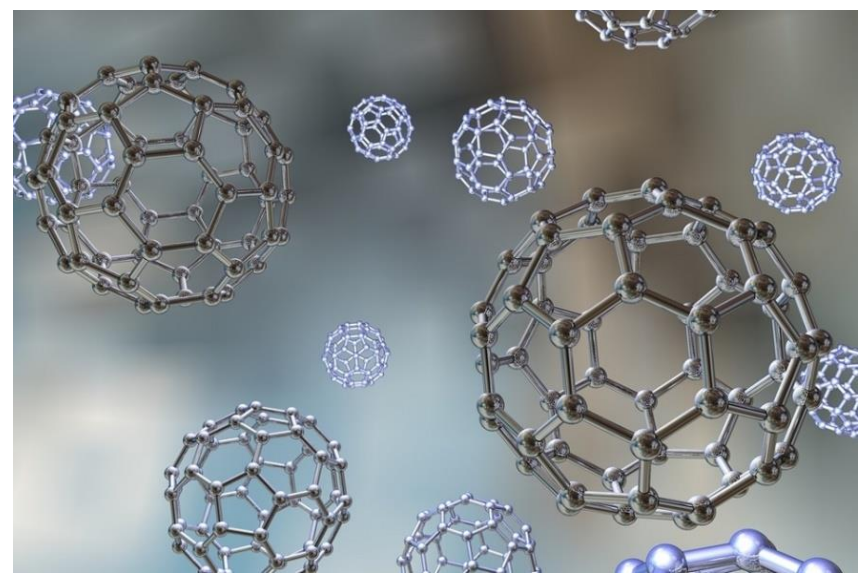

Figure 3: Nanoparticles

\section{Liposomes:}

Liposomes are potentially valuable as ocular drug delivery systems due to their simplicity of preparation and versatility in physical characteristics. However, their use is limited by instability (due to hydrolysis of the phospholipids), limited drug loading capacity, technical difficulties in obtaining sterile preparations and blurred vision due to their size and opacity. In addition, liposomes are subject to the same rapid precorneal clearance as conventional ocular solutions, especially the ones with a negative or no surface charge. Positively charged liposomes, on the other hand, exhibit a prolonged precorneal retention due to electrostatic interactions with the negative sialic acid residues of the mucin layer. There have been several attempts to use liposomes in combination with other newer formulation approaches, such as incorporating them into mucoadhesive gels or coating them with mucoadhesive polymers 22 .

\section{Niosomes:}

Niosomes are nonionic surfactant vesicles which exhibit the same bilayered structures as liposomes. In order to circumvent some of the limitations encountered with liposomes, such as their chemical instability, the cost and purity of the natural phospholipids, and oxidative degradation of the phospholipids, niosomes have been developed. Niosomes are biocompatible, biodegradable, and nonimmunogenic. They will be also shown to increase the ocular bioavailability of hydrophilic drugs significantly more than liposomes. This is due to the fact that the surfactants in the niosomes act as penetrations enhancers.

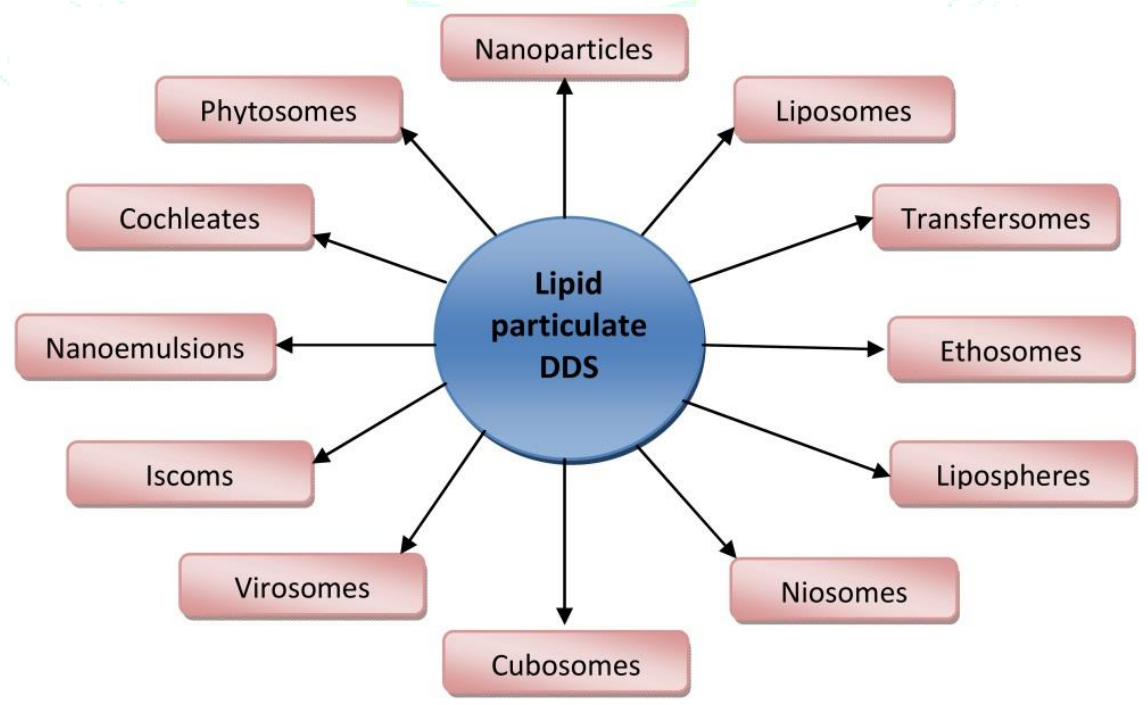

Figure 4: Lipid Nanoparticulate Drug Delivery Systems: A Revolution in Dosage Form Design and Development

\section{Microemulsions:}

Microemulsions (MEs) are colloidal dispersions composed of an oil phase, an aqueous phase, and one or more surfactants. They are optically isotropic and thermodynamically stable and appear as transparent liquids as the droplet size of the dispersed phase is less than $150 \mathrm{~nm}$. One of their main advantages is their ability to increase the solubilization of lipophilic and hydrophilic drugs accompanied by a decrease in systemic absorption (59). They can also prolong the release of drug over definite period (46). Moreover, MEs are transparent systems thus enable monitoring of phase separation and/or precipitation. In addition, MEs possess low surface tension and therefore exhibit good wetting and spreading properties. The presence of surfactants is advantageous due to an increase in cellular membrane permeability, which facilitates drug absorption and bioavailability (60). Surfactants most frequently utilized for the preparation of MEs are poloxamers, polysorbates, and polyethylene glycol derivatives (61). Caution needs to be taken in relation to the amount of surfactant incorporated, as high concentrations can lead to ocular toxicity ${ }^{23}$.

\section{Introduction to Drug ${ }^{24}$}

Ciprofloxacin is an antibiotic used to treat a number of bacterial infections. This includes bone and joint infections, intra abdominal infections, certain type of infectious diarrhea, respiratory tract infections, skin infections, typhoid fever, and urinary tract infections, among others. For some infections it is used in addition to other 
antibiotics. It can be taken by mouth, as eye drops, as ear drops, or intravenously.

Common side effects include nausea, vomiting, diarrhea and rash. Ciprofloxacin increases the risk of tendon rupture. In people with myasthenia gravis, there is worsening muscle weakness. Rates of side effects appear to be higher than some groups of antibiotics such as cephalosporins but lower than others such as clindamycin. Studies in other animals raise concerns regarding use in pregnancy. No problems will be identified, however, in the children of a small number of women who took the medication. It appears to be safe during breastfeeding. It is a second-generation fluoroquinolone with a broad spectrum of activity that usually results in the death of the bacteria.

Ciprofloxacin is patented in 1980 and introduced in 1987. It is on the World Health Organization's List of Essential Medicines, the most effective and safe medicines needed in a health system. It is available as a generic medication and is not very expensive.<smiles>O=C(O)c1cn(C2CC2)c2cc(N3CCNCC3)c(F)cc2c1=O</smiles>

Figure 5: Structure of Ciprofloxacin

\section{Medicinal uses}

Ciprofloxacin is used to treat a wide variety of infections, including infections of bones and joints, endocarditis, gastroenteritis, external respiratory tract infections, cellulitis, urinary tract infections, prostatitis, anthrax.

Ciprofloxacin only treats bacterial infections; it does not treat viral infections such as the common cold. For certain use including acute sinusitis, lower respiratory tract infections and uncomplicated gonorrhea, ciprofloxacin is not considered a first-line agent. Ciprofloxacin occupies an important role in treatment guidelines issued by major medical societies for the treatment of serious infections, especially those likely to be caused by Gram-negative bacteria, including Pseudomonas aeruginosa. For example, ciprofloxacin in combination with metronidazole is one of several first-line antibiotic regimens recommended by the Infectious Diseases Society of America for the treatment of community-acquired abdominal infections in adults. It also features prominently in treatment guidelines for acute pyelonephritis, complicated or hospital-acquired urinary tract infection, acute or chronic prostatitis, certain types of endocarditis, certain skin infections, and prosthetic joint infections 25 .

In other cases, treatment guidelines are more restrictive, recommending in most cases that older, narrower-spectrum drugs be used as first-line therapy for less severe infections to minimize fluoroquinolone-resistance development.

\section{Mechanism of action}

Ciprofloxacin is a broad-spectrum antibiotic of the fluoroquinolone class. It is active against some Grampositive and many Gram-negative bacteria. It functions by inhibiting DNA gyrase, and a type II topoisomerase, topoisomerase IV, necessary to separate bacterial DNA, thereby inhibiting cell division.

\section{Pharmacokinetics}

Ciprofloxacin for systemic administration is available as immediate-release tablets, extended-release tablets, an oral suspension, and as a solution for intravenous administration. When administered over one hour as an intravenous infusion,[28] ciprofloxacin rapidly distributes into the tissues, with levels in some tissues exceeding those in the serum. Penetration into the central nervous system is relatively modest, with cerebrospinal fluid levels normally less than $10 \%$ of peak serum concentrations. The serum half-life of ciprofloxacin is about $4-6$ hours, with $50-70 \%$ of an administered dose being excreted in the urine as unmetabolized drug. An additional $10 \%$ is excreted in urine as metabolites. Urinary excretion is virtually complete 24 hours after administration. Dose adjustment is required in the elderly and in those with renal impairment.

Ciprofloxacin is weakly bound to serum proteins (20-40\%), but is an inhibitor of the drug-metabolizing enzyme cytochrome P450 1A2, which leads to the potential for clinically important drug interactions with drugs metabolized by that enzyme. Ciprofloxacin is about $70 \%$ orally available when administered orally, so a slightly higher dose is needed to achieve the same exposure when switching from IV to oral administration ${ }^{26}$.

\section{Adverse effects}

Adverse effects can involve the tendons, muscles, joints, nerves, and the central nervous system.

Rates of adverse effects appear to be higher than with some groups of antibiotics such as cephalosporins but lower than with others such as clindamycin. Compared to other antibiotics some studies find a higher rate of adverse effects while others find no difference.

In clinical trials most of the adverse events will be described as mild or moderate in severity, abated soon after the drug is discontinued, and required no treatment. Some adverse effects may be permanent. Ciprofloxacin is stopped because of an adverse event in $1 \%$ of people treated with the medication by mouth. The most frequently reported drugrelated events, from trials of all formulations, all dosages, all drug-therapy durations, and for all indications, will be nausea $(2.5 \%)$, diarrhea $(1.6 \%)$, abnormal liver function tests $(1.3 \%)$, vomiting $(1 \%)$, and rash $(1 \%)$. Other adverse events occurred at rates of $<1 \%$ [26].

\section{Drug Delivery for Posterior Segment Eye Disease}

In recent years, significant advances have been made in optimizing the delivery of drugs to target tissues within the eye and in maintaining effective drug doses within those tissues. Most pharmacologic management of ocular disease, however, continues to use the topical application of solutions to the surface of the eye as drops. Factors that can limit the usefulness of topical drug application include the significant barrier to solute flux provided by the corneal epithelium and the rapid and extensive precorneal loss that occurs as the result of drainage and tear fluid turnover. After the instillation of an eyedrop (maximum of $30 \mu \mathrm{l}$ ) into the inferior fornix of the conjunctiva, the drug mixes with the lacrimal fluid, and drug contact time becomes a function of lacrimation, tear drainage and turnover, and to some extent the composition of the precorneal tear film itself. It has been estimated that typically less than $5 \%$ of a topically applied drug permeates the cornea and reaches intraocular tissues. The major portion of the instilled dose is absorbed systemically by way of the conjunctiva, through the highly vascular conjunctival stroma and through the lid margin 
vessels. Significant systemic absorption also occurs when the solution enters the nasolacrimal duct and is absorbed by the nasal and nasopharyngeal mucosa. Despite the relatively small proportion of a topically applied drug dose that ultimately reaches anterior segment ocular tissues, topical formulations remain effective, largely because of the very high concentrations of drugs that are administered 27.

Recent advances in topical drug delivery have been made that improve ocular drug contact time and drug delivery, including the development of ointments, gels, liposome formulations, and various sustained and controlled-release substrates, such as the Ocusert, collagen shields, and hydrogel lenses. The development of newer topical delivery systems using polymeric gels, colloidal systems, and cyclodextrins will provide exciting new topical drug therapeutics. The delivery of therapeutic doses of drugs to the tissues in the posterior segment of the eye, however, remains a significant challenge.

Currently, the treatment of posterior segment disease is to a significant extent limited by the difficulty in delivering effective doses of drugs to target tissues in the posterior eye (Fig.1) . Four approaches may be used to deliver drugs to the posterior segment-topical, systemic, intraocular, and periocular (including subconjunctival, sub-Tenon's, and retrobulbar). Topically applied drugs may enter the eye by crossing the conjunctiva and then diffusing through the sclera, ${ }^{34}$ but for reasons previously cited, this approach typically does not yield therapeutic drug levels in the posterior vitreous, retina, or choroid, and although systemic administration can deliver drugs to the posterior eye, the large systemic doses necessary are often associated with significant side effects. An intravitreal injection provides the most direct approach to delivering drugs to the tissues of the posterior segment, and therapeutic tissue drug levels can be achieved. Intravitreal injections, however, have the inherent potential side effects of retinal detachment, hemorrhage, endophthalmitis, and cataract. Repeat injections are frequently required, and they are not always well tolerated by the patient. Further, drugs injected directly into the vitreous are rapidly eliminated. Intravitreal sustainedrelease devices have been used to avoid repeated injections. The best known of these devices is the Vitrasert ganciclovir implant, used in the treatment of cytomegalovirus retinitis. ${ }^{5}$ These and other intravitreal sustained release systems, including other implant devices, microspheres, and liposomes, are exciting new modalities of drug delivery that offer effective treatment of visually devastating diseases. The devices, however, do require intraocular surgery, must be replaced periodically, and have potential side effects similar to those associated with intravitreal injection 28 .

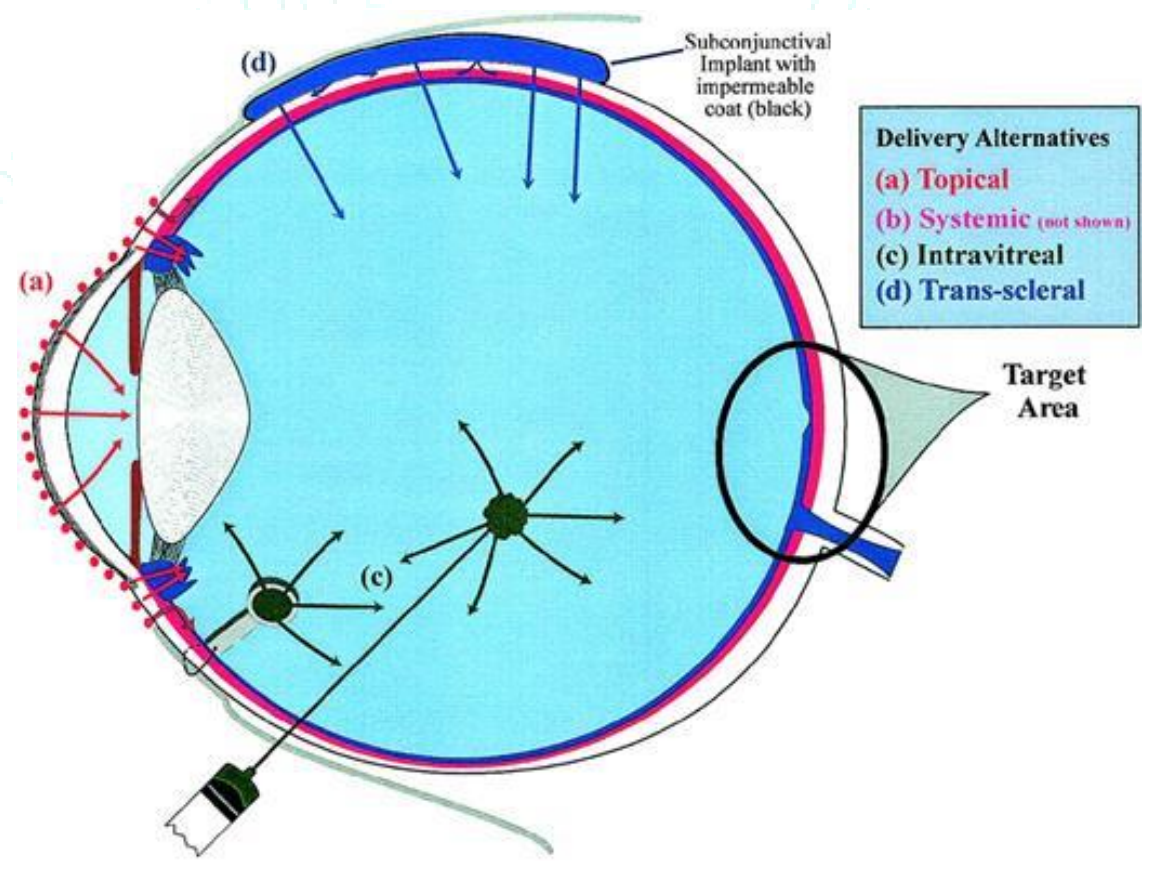

Figure 6: Delivery alternatives for Nanoparticles

Periocular drug delivery using subconjunctival or retrobulbal injections or placement of sustained-release devices provides another route for delivering drugs to the posterior tissues of the eye. This approach to drug delivery is safer and less invasive than intravitreal injection and also offers the exciting potential for localized, sustained-release drug delivery. Drug delivery by this vector ideally would be transscleral and thus could take advantage of the large surface area of the sclera. The average $17-\mathrm{cm}^{2}$ surface area of the human sclera accounts for $95 \%$ of the total surface area of the globe and provides a significantly larger avenue for drug diffusion to the inside of the eye than the 1$\mathrm{cm}^{2}$ surface area of the cornea. Also, regional differences in scleral thickness could be used to further optimize transscleral drug diffusion if sustained-release delivery devices or systems could be placed in regions where scleral permeability was greatest. The sclera, for example, is $1.0 \mathrm{~mm}$ thick near the optic nerve and an average of $0.53 \mathrm{~mm}$ thick at the corneoscleral limbus and thins to an average of 0.39 $\mathrm{mm}$ at the equator, where it can be as thin as $0.1 \mathrm{~mm}$ in a significant number of eyes. Further, an increasing body of evidence suggests that the sclera is quite permeable to a wide range of solutes and holds significant potential for posterior segment drug delivery ${ }^{29}$.

Drug delivery across the sclera or cornea is governed in part by transient diffusion across the tissue that typically occurs over a time course of minutes unless some type of controlled release formulation or device is used. Experimental measurements of scleral permeability are, however, based 
on determinations of steady state flux. It is important to note that in the absence of a sustained-release system, drugsclera contact times would be expected to be too brief to permit the attainment of steady state flux. Thus, in vitro flux measurements can be expected to over predict transscleral drug delivery. The utilization of some type of sustainedrelease delivery system would appear to be necessary for successful utilization of transscleral drug delivery. The ideal sustained-release system would provide controlled, longterm drug release, specific scleral site delivery and prolong drug-sclera contact time. This would permit improved drug flux through thinner areas of the tissue, potentially permit treatment to specific posterior segment regions, and minimize systemic drug absorption by the conjunctival vasculature. A wide variety of sustained-release drug delivery systems exist, including various gel formulations, erodible polymers, microspheres, liposomes, and various types of inserts, including miniosmotic pumps and combinations of these technologies. Two currently available technologies show exciting potential for transscleral application. In situ forming polymeric gels are viscous liquids that on exposure to physiological conditions will shift to a gel phase. Pluronic F-127 is a polyol compound that exhibits the phenomenon of reverse thermal gelation, remaining in the liquid state at refrigerator temperatures and gelling on warming to ambient or physiological temperatures. Bioadhesive compounds such as fibrin glue also hold great promise. Both Pluronic F-127 and fibrin glue have been used widely in medical and pharmaceutical systems. ${ }^{15} 16$ These compounds have excellent tissue compatibility. Drugs can be incorporated into them, and the formulation can be applied to a scleral site, where it will quickly gel or solidify. Preliminary in vitro perfusion studies with F-127 and fibrin glue have demonstrated that they can provide slow, uniform sustained release of dexamethasone across human sclera 30 .

Experimental evidence currently shows that transscleral delivery of drugs can be accomplished and suggests great promise that this approach will provide new therapeutic approaches for treating visually devastating diseases of the posterior segment of the eye. Future studies will further define the feasibility of this approach ${ }^{31}$.

\section{Acknowledgement:}

The authors are thankful to IIMT College of Pharmacy, Knowledge Park III Greater Noida, G.B Nagar for providing infrastructure and assistance towards this research work.

\section{Conflict of Interest:}

The authors declare no conflict of interest.

\section{References}

1. Pijl BJ, Theelen T, Tilanus MA, Acute Endophthalmitis After Cataract Surgery: 250 Consecutive Cases Treated at a Tertiary Referral Center in the Netherlands. American Journal of Ophthalmologist, 2010; 149:482-7.

2. Kresloff MS, Castellarin AA, Zarbin MA. 'Endophthalmitis', Survey of ophthalmology, 1998; 43(6):193-224.

3. Greenwald MJ, Principles and Practice of Pediatric Infectious Diseases. 3rd edition: Churchill Livingstone-An Imprint of Elsevier; 2008.

4. Hariprasad SM, Blinder KJ, Shah GK, et al. Determination of Vitreous and Aqueous Concentration of Topically Administered Moxifloxacin $0.5 \%$ in Humans. Investigative Ophthalmology \& Visual Science, 2004; 45:525.

5. Vidyashankar B, Arora S, Singal R, et al. Medical Treatment of Endophthalmitis. Journal of the Bombay Ophthalmologist's Association, 2001; 11:47-50.

6. Raichand M, Endophthalmitis: Diagnosis and management
Indian Journal of Ophthalmology, 1982; 30(6):635-40.

7. Mah FS, The fourth-generation fluoroquinolones: New topical agents in the war on ocular bacterial infections. Current Opinion in Ophthalmology, 2004; 15(4):316-20.

8. Gardner S. The Evolution of Modern Fluoroquinolones, Advanced Ocular Care, 2010; (4).

9. Ogura $Y$, Drug delivery to the posterior segments of the eye. Advanced Drug Delivery Reviews, 2001;52:1-3.

10. Jacquin C, Nasica X, Guillard D, et al, Intravitreal treatment of endophthalmitis. Méd Mal Infect- France, 2001; 31:49397.

11. Armaly MF, Rao KR. The effect of pilocarpine Ocusert with different release rates on ocular pressure. Investigative Ophthalmology. 1973; 12:491-96.

12. Cordonnier M, The treatment of dry eye with Lacrisert. Bull Soc Belge Ophtalmol, 1984; 212:65-9.

13. Del Amo EM, Urtti A, Current and future ophthalmic drug delivery system- A shift to the posterior segment. Drug Discovery Today, 2008; 13(2).

14. Rupenthal ID, Alany RG, Pharmaceutical manufacturing handbook - Production and processes. Wiley-Interscience, 2008.

15. Jarvinen $\mathrm{K}$, Jarvinen $\mathrm{T}$, Ocular absorption following topical delivery. Advanced Drug Delivery Reviews, 1995;16:3-19.

16. Worakul N, Robinson JR, Ocular pharmacokinetics/ pharmacodynamics: Review. European Journal of Pharmaceutics and Biopharmaceutics, 1997; 44(1627):7183.

17. Vellonen KS, Mannermaa E, Urtti A, Drug transport in corneal epithelium and blood-retina barrier: Emerging role of transporters in ocular pharmacokinetics. Advanced Drug Delivery Reviews, 2006; 58:1136-1163.

18. Macha S, Hughes PM, Mitra AK, Ophthalmic Drug Delivery Systems, 2nd edition, Marcel Dekker, 2003.

19. Huang AJW, Tseng SCG, Kenyon KR, Paracellular permeability of corneal and conjunctival epithelia Investigative Ophthalmology and Visual Science, 1989; 30(4):684-9.

20. Greaves JL. Wilson CG. Treatment of diseases of the eye with mucoadhesive delivery systems. Advanced drug delivery review, 1993; 11(3): 349-383.

21. Ahmed I, Patton TF. Importance of the noncorneal absorption route in topical ophthalmic drug delivery. Investigative Ophthalmology and Visual Science, 1985; 26(4):584-7.

22. Urtti A, Challenges and obstacles of ocular pharmacokinetics and drug delivery. Advanced Drug Delivery Reviews, 2006; 58:1131-1135.

23. Lee $\mathrm{VH}$, New directions in the optimization of ocular drug delivery. Journal of Ocular Pharmacology, 1990; 6(2):15764.

24. Shinoda K, Lindman B, Organised surfactant systems: microemulsions, Langmuir, 1987; 3:135-49.

25. Sushama T, Adnan A, Farhan JA, Microemulsion: A Novel Approach to Enhanced Drug Delivery. Recent Patents on Drug Delivery \& Formulation, 2008; 2:238-57.

26. Anna RS, Joanna W, Microemulsions as Potential Ocular Drug Delivery Systems: Phase Diagrams and Physical Properties.

27. Sumedha N, Chandrakant L, Current Trends in Novel Drug Delivery An OTC Perspective. Pharma Times, 2010; 42:1723.

28. Vandamme TF, Microemulsions as ocular drug delivery systems: recent developments and future challenges. Progress in Retinal and Eye Research, 2002; 21:15-34.

29. Kumar K, Senthil D, SaravananR, Microemulsions as Carrier for Novel Drug Delivery: A Review. International Journal of Pharmaceutical Sciences Review and Research, 2011; 10:37-45.

30. Peltola S, Saarinen P, Kiesvaara I, Microemulsions for topical delivery of estradiol, Int. J. Pharm. 2003; 254:99107

31. Huabing C, Xueling C, Danrong D, Microemulsion-based hydrogel formulation of ibuprofen for topical delivery. International Journal of Pharmaceutics, 2006; 351: 52-58. 